\title{
AEROELASTIC ANALYSIS OF MODERN COMPLEX WINGS
}

\author{
Rakesh K. Kapania and Manoj K. Bhardwaj"* \\ Virginia Polytechnic Institute and State University \\ Blacksburg, VA 24060-0203 \\ Eric Reichenbach ${ }^{\dagger}$ \\ McDonnell Douglas Aerospace \\ St. Louis, Missouri 63166 \\ Guru P. Guruswamy \\ Computational Aerosciences Branch \\ NASA Ames Research Center, Moffett Field, CA 94035-1000
}

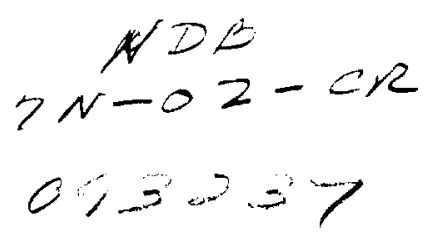

\begin{abstract}
A process is presented by which aeroelastic analysis is performed by using an advanced computational fluid dynamics (CFD) code coupled with an advanced computational structural dynamics (CSD) code. The process is demonstrated on an F/A-18 Stabilator using NASTD (an in-house McDonnell Douglas Aerospace East CFD code) coupled with NASTRAN. The process is also demonstrated on an aeroelastic research wing (ARW-2) using ENSAERO (an in-house NASA Ames Research Center CFD code) coupled with a finite element wing-box structures code. Good results have been obtained for the F/A-18 Stabilator while results for the ARW-2 supercritical wing are still being obtained.
\end{abstract}

\section{Introduction}

Aeroelastic analysis requires solutions of both the fluid and structural equations together. Both uncoupled and coupled methods exist in solving these non-linear system of equations'. The less expensive uncoupled methods can only handle small perturbations with moderate non-linearity. Aeroelastic problems involve aerospace vehicles with large structural deformations and highly non-linear characteristics, therefore fully coupled methods are needed to solve these aeroelastic problems accurately.

Fully coupled methods require interface procedures

\footnotetext{
'Professor, Aerospace and Ocean Engineering, Associate Fellow AIAA

"Graduate Research Assistant, Aerospace and Ocean

Engineering, Student Member AIAA

'Senior Project Engineer, Member AIAA

'Research Scientist, Associate Fellow AIAA
}

which introduce a certain level of complexity. This complexity is related to the level of fluid and/or structural model used. Recently the Virginia Tech Multidisciplinary Analysis and Design (MAD) Center Advisory Board met to discuss the needs of industry in performing multidisciplinary analysis. There was expressed a strong need for a robust interface process that will allow a coupling of two independent codes, specifically a fluids analysis code and a structural analysis code, to perform aeroelastic analysis. And with advanced subsonic transports entering into the transonic regime and fighter aircraft being limited by aeroelastic phenomena, it is becoming increasingly important to perform static and dynamic aeroelastic analysis using highly accurate fluid and structural models.

Work has been done coupling the fluid and structural domains to perform static aeroelastic analysis. Euler flow equations coupled with finite element wing-box structures using a simple interface procedure between the two domains is used to perform static aeroelastic analysis as can be seen in ref. 2 . Also, recently Euler flow equations coupled with finite element wing-box structures using better triangular elements than the study in reference 1 are used to perform static aeroelastic analysis exploiting parallel computers (ref. 3). Again, the interface procedure was a simple one. Other methods involving the coupling of the fluid and structural domains can also be seen in ref. 4 .

In this paper, a process is presented by which static aeroelastic analysis is performed using highly detailed computational fluid dynamics (CFD) and highly detailed computational structural dynamics (CSD). The process deals with the interfacing of two separate codes in the CSD and CFD fields. 
Aeroelastic Coupling Procedure

The process by which static aeroelastic analysis is to be performed is broken down into the following steps:

1) Get CFD solution

2) Calculate pressures at CFD grid points on aerodynamic surface

3) Map pressures on CFD grid to forces on CSD grid

4) Obtain response of the structure

5) Map displacements on CSD grid to

displacements on aerodynamic surface of the CFD

grid

6) Deform entire CFD grid

7) Repeat steps until convergence criteria is met

The above steps will sometimes be referred to later as one cycle.

When obtaining the CFD solution, it need not be converged completely in the first few cycles if starting from free stream boundary conditions, since the convergence of the aeroelastic process is usually oscillatory. But, to converge the aeroelastic process in fewer cycles, it is better to obtain the rigid steady state solution before beginning the first cycle of the aeroelastic analysis process. This will help reduce the computational time if structural model will be changed often.

After the pressures on the aerodynamic surface of the CFD grid are calculated, they are mapped from the CFD grid to forces on the CSD grid. This involves a preprocessed mapping. The mapping consists of the following information. For each CFD point $(i, k)$ on the aerodynamic surface, the area on which the pressure acts and unit normal is calculated. Now, the magnitude and direction of the force due to unit pressure are known. The next step is to find a structural triangle that surrounds the CFD point $(i, k)$. This can be difficult due to the irregular grids of some structural models.

It is assumed that the structural grid is divided into an upper and lower surface structural grids with overlapping points possibly occurring at the leading and trailing edges and also at the tip. To find the structural triangle associated with the CFD grid point, the 20 closest structural nodes are found using the upper or lower surface structural grid depending on which surface the CFD point is located. Then all possible triangles using the 20 points are formed. Next, the triangles that do not contain the CFD point $(\mathrm{i}, \mathrm{k})$ as an interior point are eliminated. Then of the remaining triangles, the largest vertex distance is measured for each triangle, where vertex distance is the distance between the structural node and CFD point $(i, k)$. Finally, the triangle with the smallest largest vertex distance is chosen. Now that the structural triangle is known, the area coordinates of the CFD point are used to distribute the force to the nodes of the structural triangle. So for each CFD point $(i, k)$, the necessary weight factors and destination nodes are known in the preprocessing stage as well as the direction of the application of the loads. As a side note, the 20 closest points can be changed to 25 closest points depending on the density of the structural grid.

Now that the forces on the CSD grid are known, the structural response of the system is calculated. This is done by solving the following system of equations $[K]\left\{u_{s}\right\}=\left\{f_{s}\right\}$. This can be done easily by any structural analysis tool to obtain the displacements, $\left\{u_{s}\right\}$, on the CSD grid.

Once the structural response, $\left\{u_{s}\right\}$, is known, the displacements, $\left\{u_{a}\right\}$, on the aerodynamic portion of the CFD grid need to be calculated. This is done by using a surface spline $e^{3}$. The surface spline system of equations become $[A]\{c\}=\left\{u_{\text {spl }}\right\}$ where $[A]$ is dependent on the coordinates of the spline points, $\{c\}$ is the vector of unknown coefficients of the surface spline equation, and $\left\{u_{s p p}\right\}$ are the displacements at the spline points. In the preprocessing stage, some of the structural nodes are chosen as the spline points. Once the spline points are chosen, $[A]$ is formed using the coordinates of the spline points. After the structural response, $\left\{u_{s}\right\}$, is obtained, the spline point displacements, $\left\{u_{\text {spl }}\right\}$, are extracted, and $\{c\}$ is calculated. Now, the displacements on the aerodynamic surface portion of the CFD grid, $\left\{u_{2}\right\}$, are calculated using the coordinates of the CFD grid points.

Now that $\left\{u_{a}\right\}$ are known, the next step is to deform the entire CFD volume grid. This is dependent on the fluid analysis tool. In this study, two separate codes for fluid analysis are used. One of the codes, ENSAERO, has a built in scheme to move the grid, once the surface grid is deformed. The other code used, NASTD, does not have a scheme to move the grid. So a simple grid moving scheme is applied to the case when NASTD is used, which is dependent on the CFD grid of the aerodynamic surface. It simply uses the deflections calculated, $\left\{u_{a}\right\}$, and moves the aerodynamic surface the same amount. The remaining grid is deformed in the normal direction using a spacing function that varies smoothly from 1 to 0 . This will be discussed more when describing the specific example used. 
First, the process is demonstrated by using Euler flow equations in NASTD (an in-house McDonnell Douglas Aerospace East CFD code) and an advanced structural analysis tool, NASTRAN. The F/A-18 Stabilator (horizontal tail) is used to demonstrate the process, and results have been acquired for this example.

Next, the process is demonstrated by using Euler flow equations in ENSAERO ${ }^{6,7}$ (an in-house NASA Ames Research Center CFD code) and a finite element wingbox structural model. A supercritical wing (ARW-2) is chosen to demonstrate the process. Results are being obtained for this example.

\section{E/A-18 Stabilator}

As mentioned earlier, the F/A-18 Stabilator is chosen to demonstrate the process using Euler flow equations as used in NASTD at sea-level, one degree angle of attack, Mach 0.95. The CFD grid of the F/A-18 Stabilator, approximately 800,000 grid points, is shown in figure 1. NASTRAN is used to analyze the structure. The stiffness matrix produced by NASTRAN is used to get the displacements given the loads, therefore during the aeroelastic analysis process, NASTRAN is not directly involved, since the stiffness matrix does not change during the process The finite element model of the F/A-18 Stabilator is shown in figure 2 which consists of approximately 2000 nodes, 12000 d.o.f. So step 1 involves getting the CFD solution. For this case, the rigid steady state solution is obtained before the aeroelastic analysis cycle begins. Once the CFD solution is obtained, the forces on the CSD grid are calculated using the preprocessed mapping. The mapping of the CFD points to the structural triangles as discussed before is graphically shown in figure 3 .

Now that the forces on the CSD grid are known, NASTRAN is used to obtain the structural response. Next, the displacements at the spline points are extracted. The spline points for this case are shown in figure 4. The reason for this choice can be seen when looking at figure 5, which is the surface grid of the F/A-18 Stabilator. The surface grid includes the aerodynamic surface and the points extending beyond the wing tip in the spanwise direction, and the points extending beyond the trailing edge in the chordwise direction. The points not on the aerodynamic surface are chosen so the displacements vary smoothly from the aerodynamic surface to the farfield. So, using the preprocessed mapping the surface spline coefficients are solved, and the deflections on the CFD surface grid are calculated.
Next, the remaining grid is deformed. In this case, the $i$ index varies circumferentially around the wing section, $\mathrm{j}$ index varies in the normal direction, and $\mathrm{k}$ index varies along the span. Once the surface deflections are known, a cosine spacing function is used to deform the grid at each $\mathrm{k}=$ constant face. The spacing function is dependent on the normal index $\mathrm{j}$. The outer boundaries of the grid do not move. This is done to take advantage of distributed computing capabilities in the future, where the CFD grid can be broken down into multiple zones. In this case the CFD grid is broken into two zones, but distributed computing was not used. After the grid is deformed, the cycle is repeated until some convergence criteria is met. As a note, the number of iterations for the convergence of the CFD solution varied during each cycle. No exact number of iterations were used for each cycle, but the difference between the iterations per cycle was minimal.

\section{ARW-2 Supercritical Wing}

The next case involves the ARW-2 Supercritical Wing. As mentioned earlier, Euler equations as demonstrated by ENSAERO are used to obtain the CFD solution. While structural analysis is done using a finite element wing-box structures code. The finite element wing-box model is created using axial bars in conjunction with Allman's triangular element ${ }^{9}$, which is a nine d.o.f. element with two in-plane translations and an in-plane rotation at each node. The element can represent all constant strain states exactly, thus assuring convergence with consistent mesh refinement.

The solver for structural domain is a direct solver

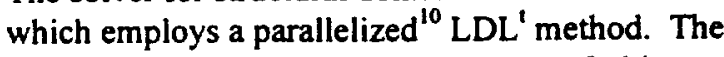
stiffness matrices are stored in a skyline fashion reducing storage requirements. This is done so that the power of parallel computing as it applies to aeroelastic analysis can be exploited in the very near future.

The CFD grid of the ARW-2 supercritical wing is shown in figure 6 , which consists of approximately 400,000 grid points. The finite element wing-box model is shown in figures 7 and 8 . Figure 7 shows the entire wing as it is discretized, while figure 8 shows the spars and ribs of the structure. The finite element model consists of approximately 400 nodes, 2400 d.o.f.

After the CFD solution of the ARW-2 supercritical wing is obtained, the next step is to use the mapping to transfer the pressures on the CFD grid to forces on the CSD grid. The mapping of the CFD grid points to the CSD grid is shown in figure 9. Once the forces on the 
structure are known, the displacements are easily solved by using the finite element wing-box code.

Once the displacements on the CSD grid, $\left\{u_{s}\right\}$ are known, the spline point displacements $\left\{u_{\text {spl }}\right\}$ are extracted. The spline points used for the ARW-2 supercritical wing are shown in figure 10 . So the surface spline system of equations is solved and the coefficients of the surface spline equation are known. Now the CFD surface grid is deformed using the surface spline equation.

Once the CFD surface grid is deformed, the next step is to deform the entire CFD volume grid. ENSAERO already has an algebraic grid moving scheme which was used in this example (Ref. 4). Therefore, once the CFD surface grid is deformed, the process of deforming the entire CFD volume grid is done using the CFD code itself.

\section{Results}

\section{E/A-18 Stabilator}

The convergence of the CFD solution is shown in figure 11. The spikes coincide with each cycle. The convergence of the structural analysis is shown in figure 12 . Here the deflections at the trailing edge of the F/A-18 Stabilator are plotted after each cycle to show the oscillatory convergence. Figure 13 shows the convergence at the trailing edge tip. As can be seen, good convergence is obtained, and the deformed and undeformed F/A-18 Stabilator is shown in figure 14. The pressure coefficient variation of the flexible versus the rigid F/A-18 Stabilator is shown in figure 15, and similarly the Mach number variation is shown in figure 16. As can be seen, the deflections are not large due to the low angle of attack of the analysis.

\section{ARW-2 Supercritical Wing}

Results are still being obtained for the ARW-2 supercritical wing. One of the problems is to develop an equivalent isotropic wing that closely matched the original ARW-2 supercritical wing which contains composite skins. To validate the isotropic ARW-2 wing, a tip load of $100 \mathrm{lb}$ in the downward direction is applied, and the deflections compared. The deflections along the front, rear, and auxiliary spars are compared and are in good agreement. Figure 17 shows the displacements plotted along the span of the rear spar. As can be seen, good agreement is obtained.
The aeroelastic coupling process has been successfully demonstrated using the F/A-18 Stabilator, while results for the ARW-2 supercritical wing are still being obtained. It was shown that the process, though somewhat problem dependent, is robust in coupling an advanced CFD tool with an advanced CSD tool. The coupling is done before the aeroelastic process is begun by creating mappings which transfer pressures on the CFD grid to forces on the CSD grid and the resulting displacements on the CSD grid back to the CFD grid to deform it. The mappings require only the CFD surface grid and CSD grid point coordinates. In addition, some user interface is required in choosing surface spline points from the CSD and CFD grids, thus creating the problem dependency. Overall, the aeroelastic coupling process has been successful.

\section{References}

1. Guruswamy, G.P. and Yang, T.Y., "Aeroelastic Time-Response Analysis of Thin Airfoils by Transonic Code LTRAN2", Computers and Fluids, Vol. 9, No. 4, Dec. 1980, pp. 409-425.

2. Macmurdy, D.E., Guruswamy, G.P., and Kapania, R.K., "Static Aeroelastic Analysis of Wings Using Euler/Navier-Stokes Equations Coupled with Improved Wing-Box Finite Element Structures", AIAA Paper 941587, April 1994.

3. Bhardwaj, M.K., Kapania, R.K., Byun, C., and Guruswamy, G.P., "Parallel Aeroelastic Computations By Using Coupled Euler Flow and Wing-Box Structural Models", AlAA Paper 95-1291, April 1995.

4. Byun, C. and Guruswamy, G.P., "Wing-Body Aeroelasticity Using Finite-Difference Fluid/Finite Element Structural Equations on Parallel Computers", AIAA Paper 94-1487, April 1994.

5. Harder, R.L. and Desmarais, R.N., "Interpolation Using Surface Splines", Journal of Aircraft, Vol. 9, No. 2, October 1971, pp. 189-191.

6. Guruswamy, G.P., "Coupled FiniteDifference/Finite Element Approach for Wing-Body Aeroelasticity", AIAA Paper 92-4680, Sep. 1992.

7. Guruswamy, G.P. and Byun, C., "Fluid-Structural Interactions Using Navier-Stokes Flow Equations Coupled with Shell Finite Element Structures", $A I A A$ Paper 93-3087, July 1993.

\section{Conclusions}


8. Sanford, Maynard C., Seidel, David A., Eckstrom, Clinton V., and Spain, Charles V., "Geometrical and Structural Properties of an Aeroelastic Research Wing (ARW-2)", NASA TM 4110, April 1989.

9. Allman, D.J., "A Compatible Triangular Element Including Vertex Rotations for Plane Elasticity Problems", Computers and Structures, Vol. 19, No. 12, 1984, pp. 1-8.

10. Farhat, C. and Wilson, E., "A Parallel Active Column Equation Solver", Computers and Structures, Vol. 28, No.2, 1988, pp. 289-304.

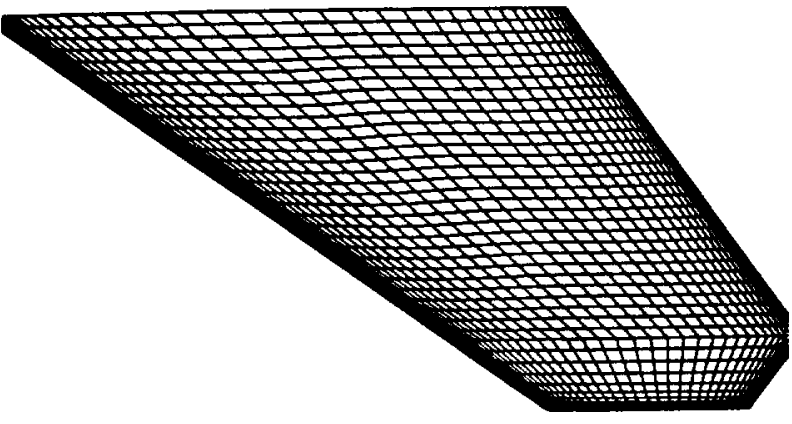

Figure I - CFD grid of F/A-18 Stabilator

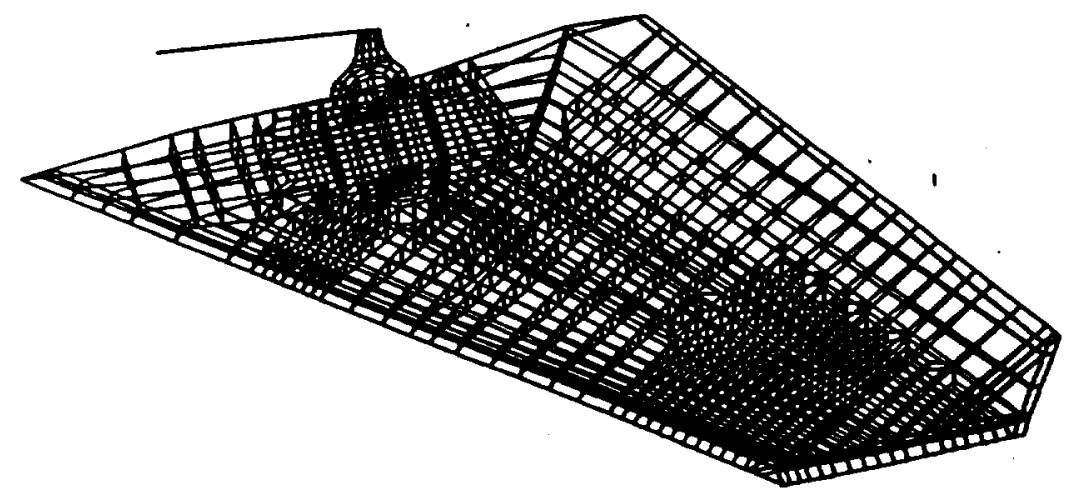

Figure 2 - F/A-18 Stabilator Finite Element Model

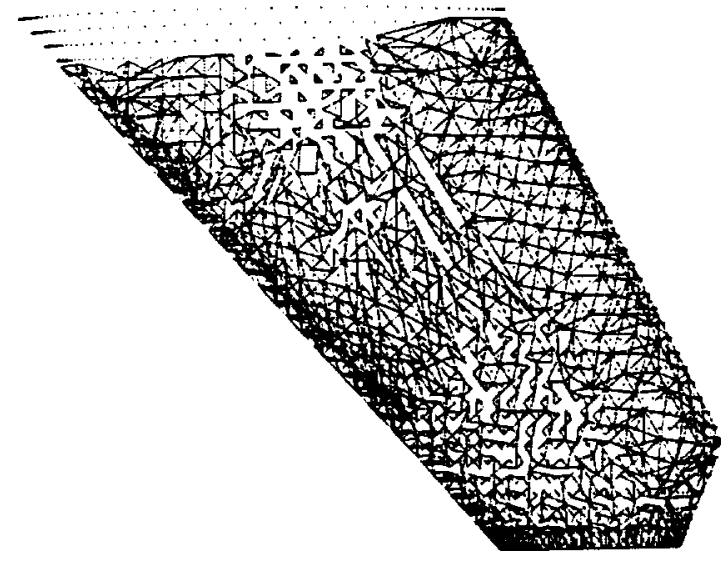

Figure 3 - Mapping of CFD points to structural triangles for F/A-18 Stabilator

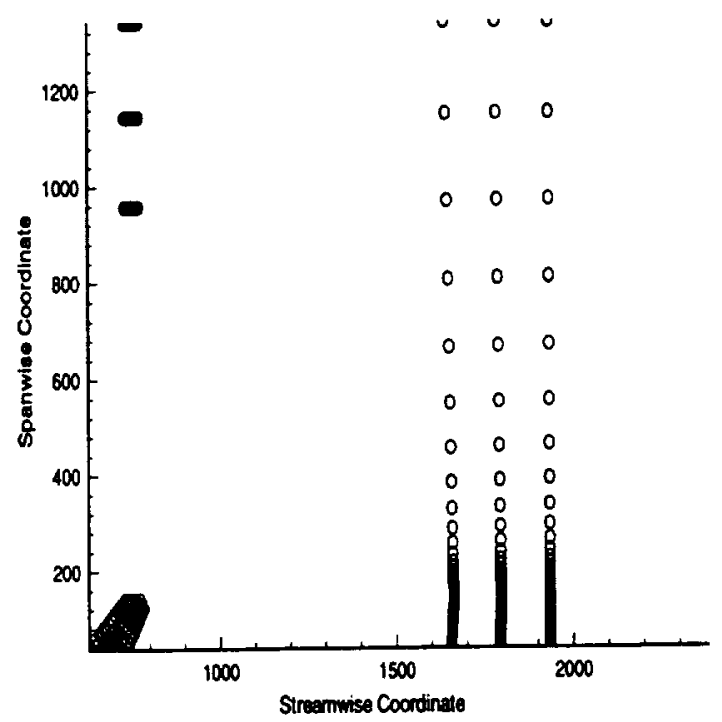

Figure 4 - Spline points used for F/A-18 Stabilator

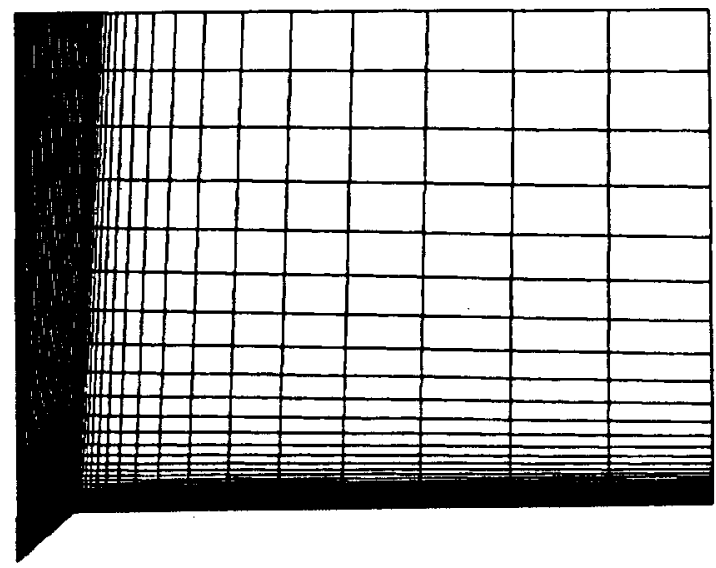

Figure 5 - Entire surface grid of F/A-18 Stabilator 


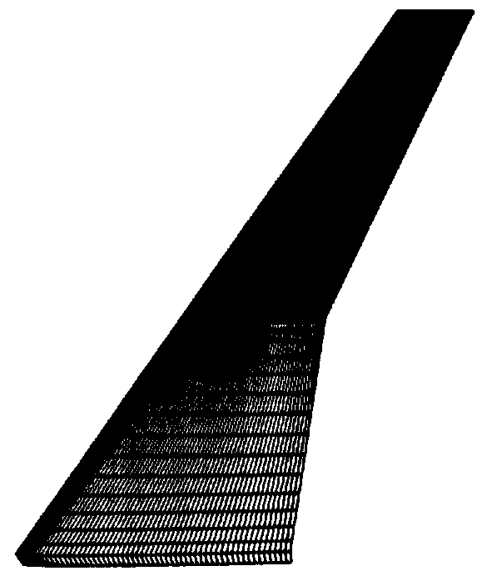

Figure 6 - CFD grid of ARW-2 supercritical wing

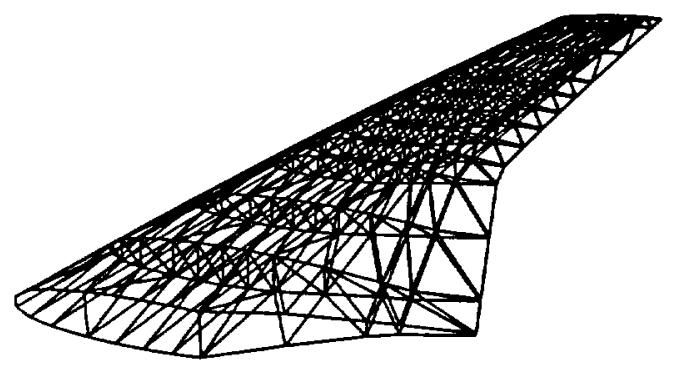

Figure 7 - Entire ARW-2 supercritical wing finite element model

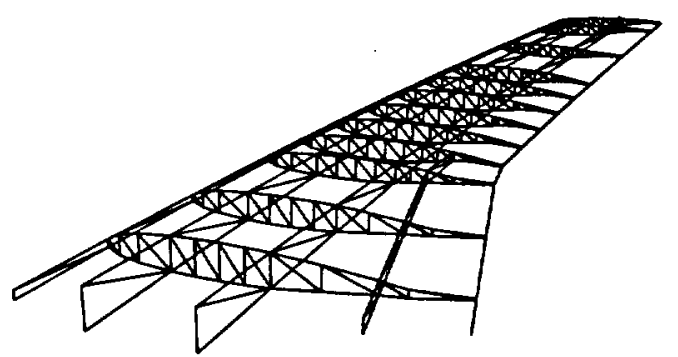

Figure 8 - Spars and ribs of ARW-2 supercritical wing

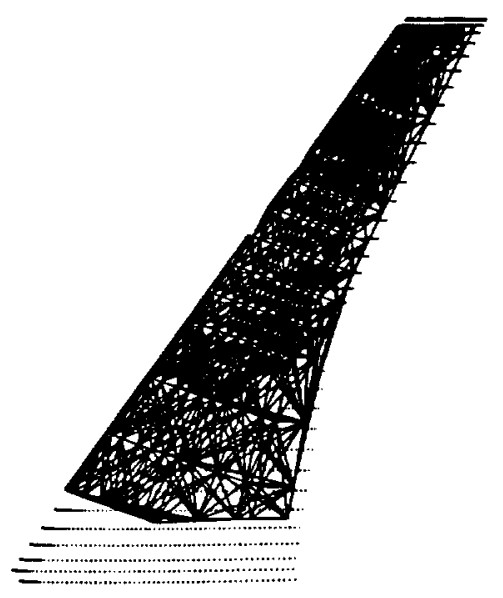

Figure 9 - Mapping of CFD grid points to structural triangles for ARW-2 supercritical wing

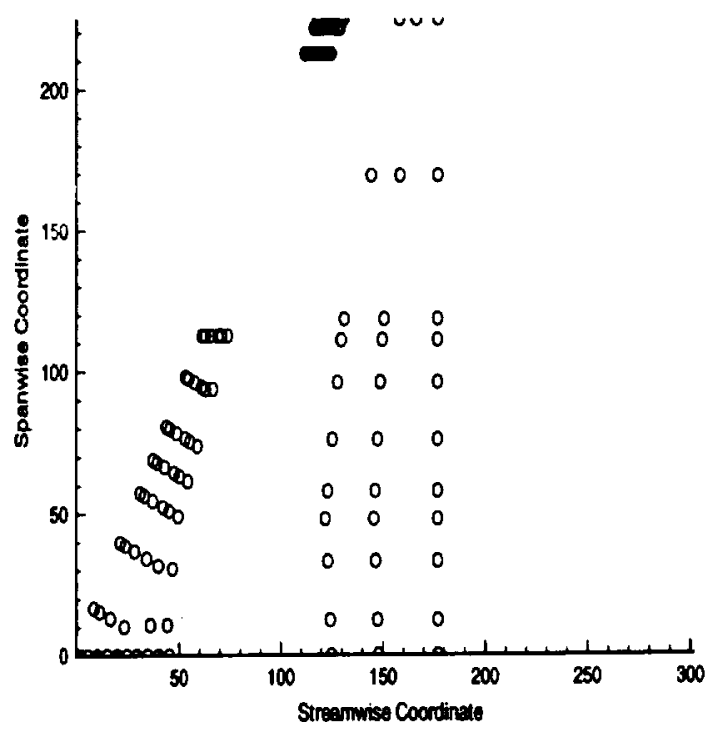

Figure 10 - Spline points used for ARW-2 supercritical =-... wing

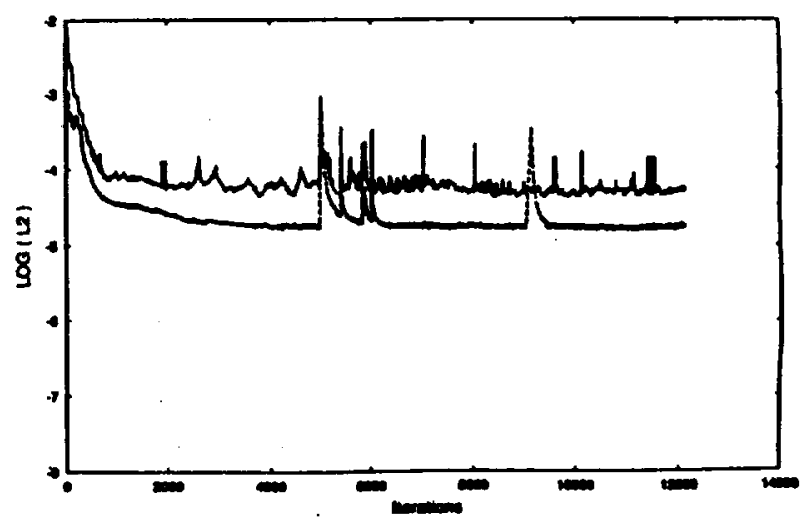

Figure 11 - Convergence of CFD solution of F/A-18 Stabilator 


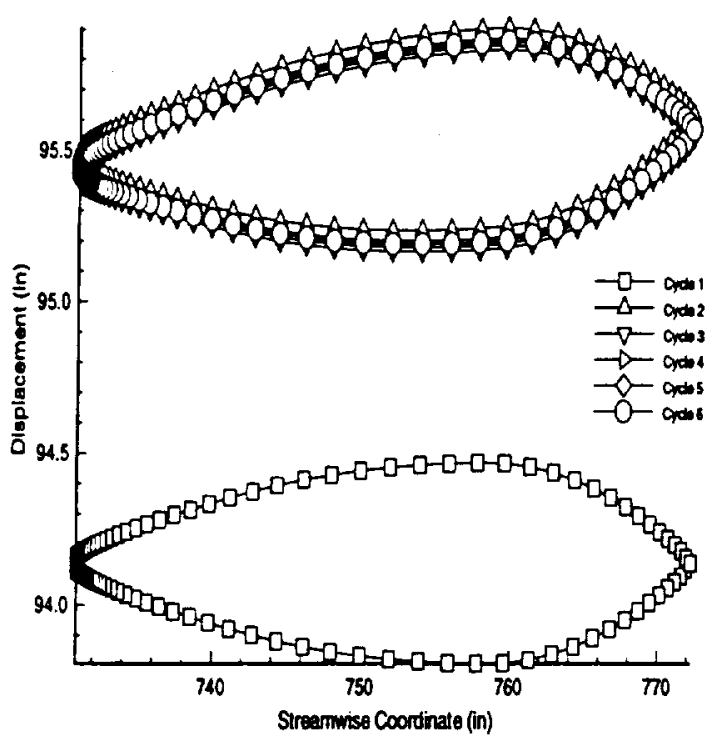

Figure 12 - Convergence of structural analysis for the F/A-18 Stabilator

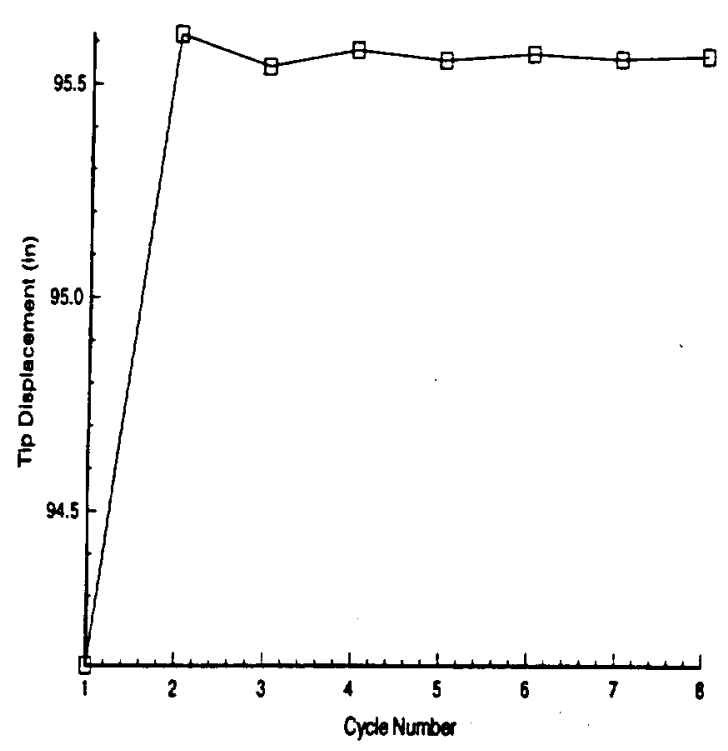

Figure 13 - Convergence of the trailing edge tip of the F/A-18 Stabilator

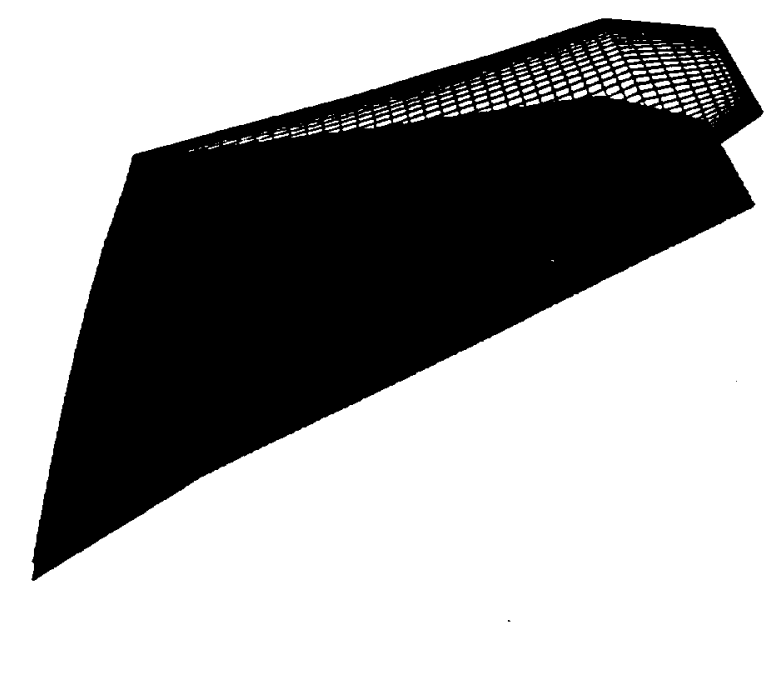

Figure 14 - Deformed and undeformed aerodynamic surfaces of the F/A-18 Stabilator
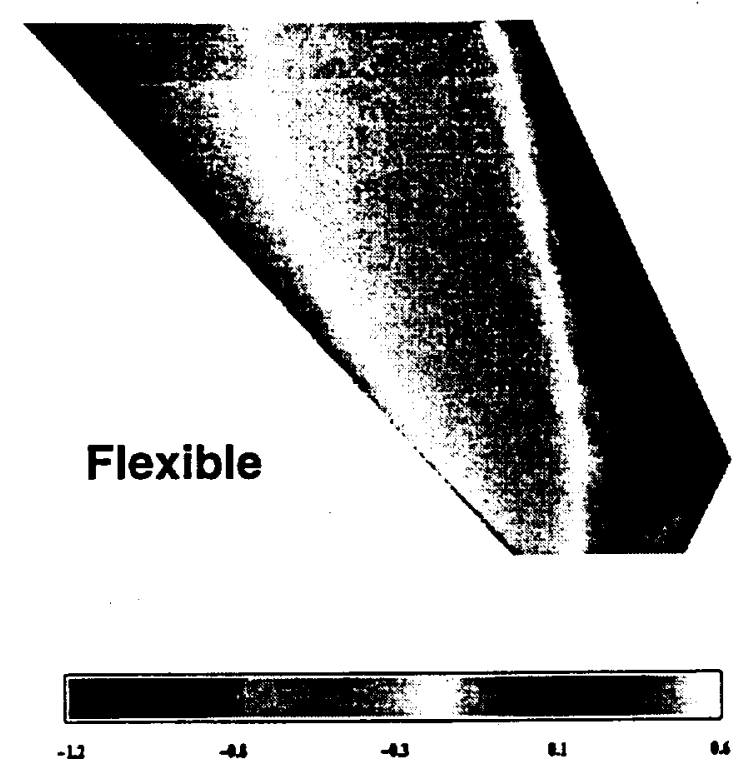

Rigid

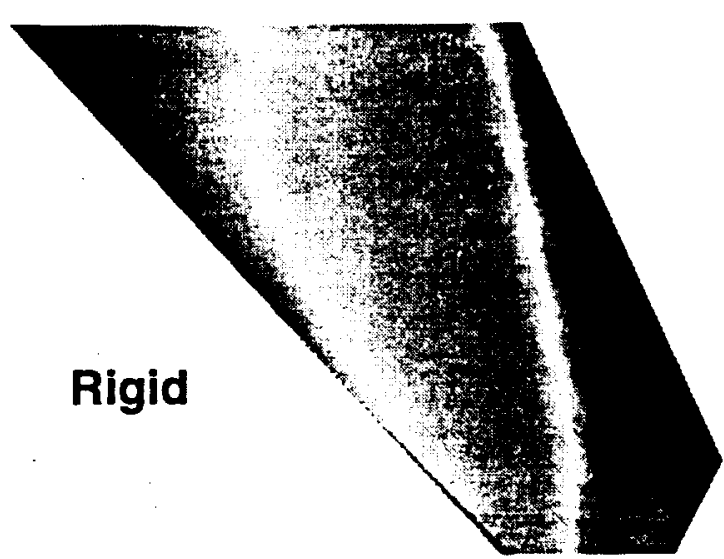

Figure 15 - Pressure coefficient variation of flexible and rigid F/A-18 Stabilator 

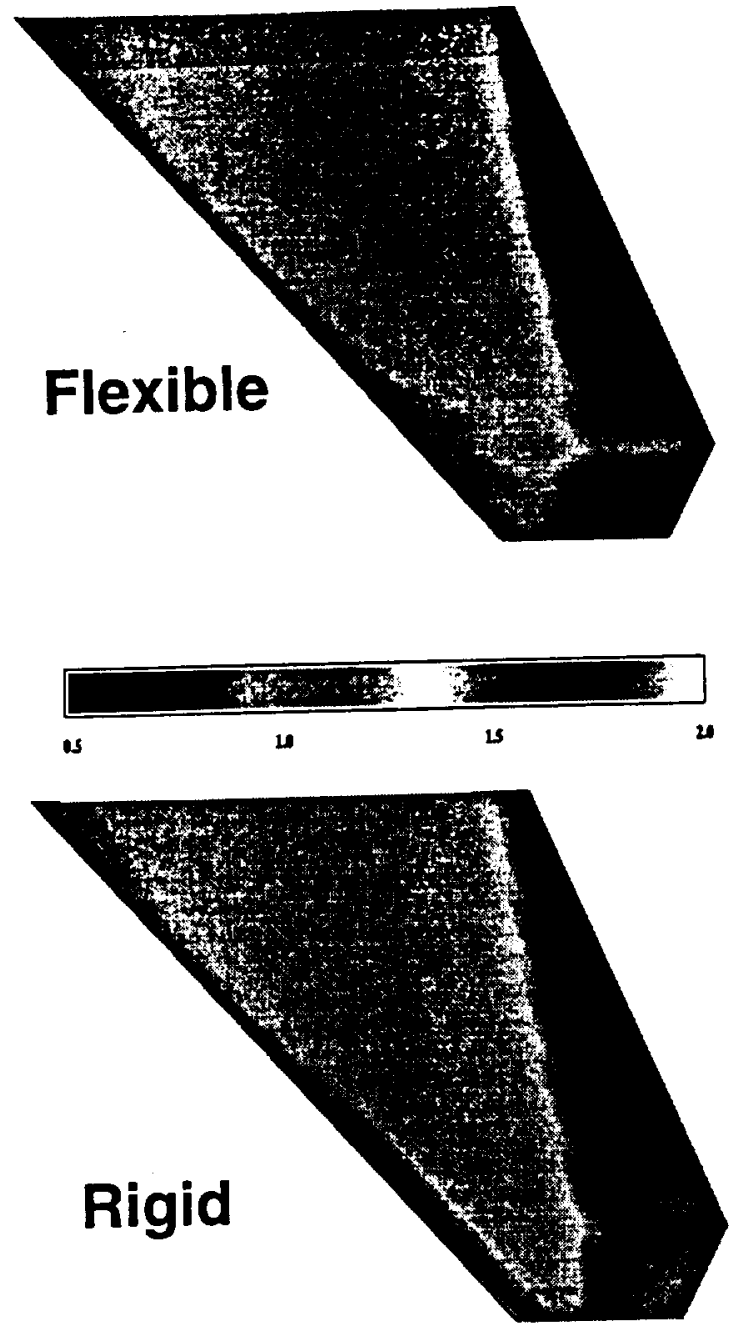

Figure 16 - Mach number variation of flexible and rigid F/A-18 Stabilator

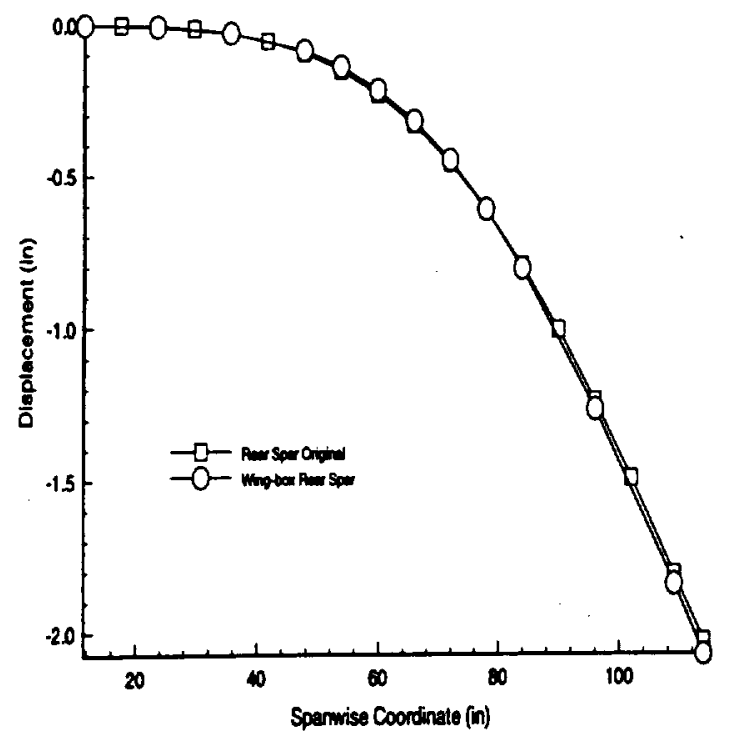

Figure 17 - Comparison of rear spar displacements of composite and isotropic ARW-2 supercritical wing 